\title{
Effects of Ecstasy on the Nervous System
}

\author{
Amirreza Katebi ${ }^{1}$, Yasmina Katebi ${ }^{2}$, Fereshte Golab ${ }^{3^{*}}$ \\ ${ }^{1}$ Department of Psychology, Faculty of Psychology and Educational Sciences, Alame Tabatabaee University, Tehran, Iran \\ ${ }^{2}$ Farzanegan 1 High School, Tehran, Iran \\ ${ }^{3}$ Cellular and Molecular Research Center, Iran University of Medical Sciences, Tehran, Iran
}

\begin{tabular}{lll}
\hline & Article Info: & \\
\hline Received: 26 Dec 2016 & Revised: 24 Jan 2017 & Accepted: 26 Jan 2017
\end{tabular}

\section{A BSTRACT}

Introduction: Ecstasy is a strong hallucinogenic drug, which is widely used as a street drug. It can improve mood and social relationships in the short term. Studies in humans and animals have shown that ecstasy can cause toxic effects on the nervous system. Although many research has been done on ecstasy but its mechanism of action is still not fully understood. Ecstasy is capable to inhibit serotonin in axon terminals of the brain in rats and mice. It destroyed neurons in the hippocampus, basal ganglia, insula, and parietal cortex. Heat gain, high blood pressure, arrhythmia, liver dysfunction, seizures, memory loss as well as cognitive and mood disorders are among toxic effects of this drug. There are some reports of its toxic effects on the nervous system. Evidence suggest that it can cause resistant psychotic disorders and depression in some consumer. Investigations have shown that the major cause of complications of this drug is reduction of serotonergic system activities. Frequent use of ecstasy (or its addiction) can cause the loss and degradation of the serotonergic neurons. Conclusion: Use of ecstasy is increasing and prevention of its damage to nervous system should be considered. Administration of neuroprotective substances may prevent these complications.

Key words:

1. Brain

2. Serotonin

3. Depression

*Corresponding Author: Fereshte Golab

E-mail: Fgolab520@gmail.com 


\title{
اثرات اكستازى بر سيستهم عصبى
}

\author{
امير رضا كاتبى'، ياسمينا كاتبى '، فرشته كلاب"

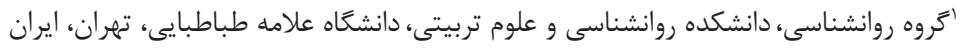

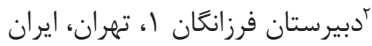 \\ 'مركز تحقيقات سلولى و مولكولى، دانشكاه علوم يزشكى ايران، تهران، ايران، ايران
}

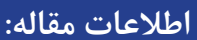

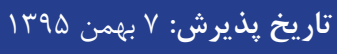

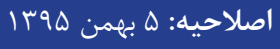

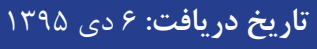

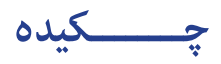

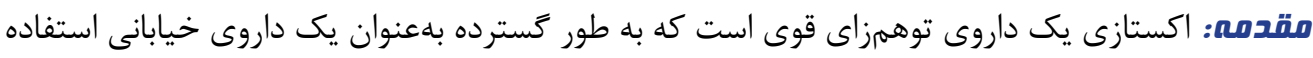

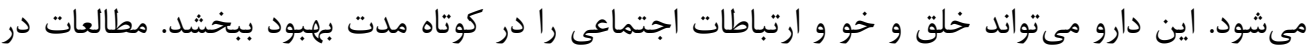

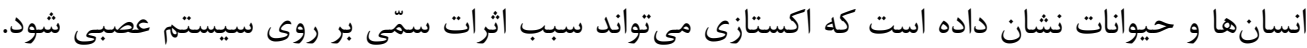

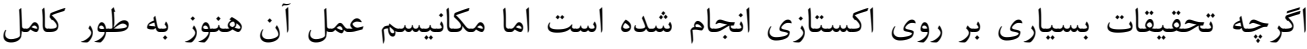

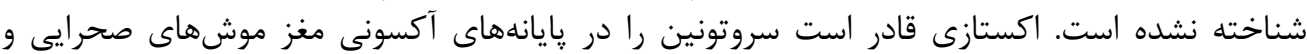

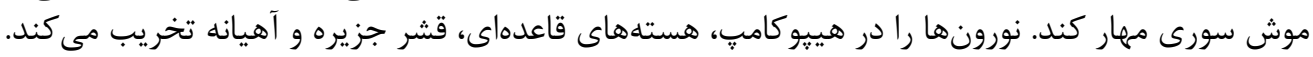

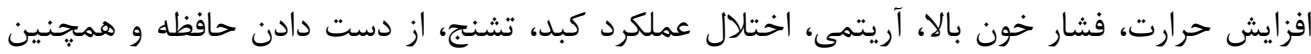

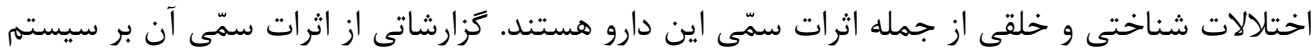

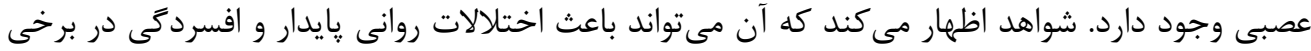

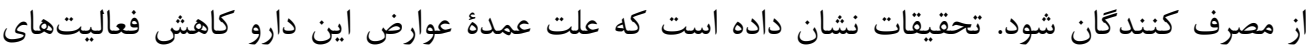

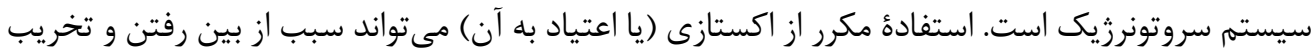

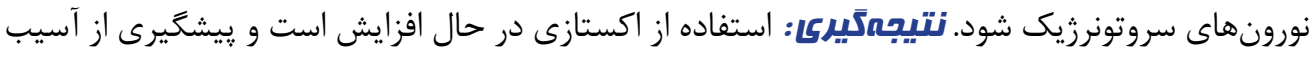

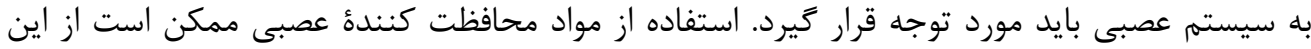
عوارض جلوگيرى كند.

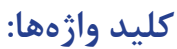

ا 1

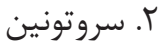

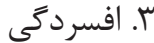

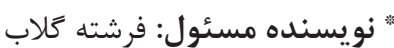

آدرس الكترونيكى: Fgolab520@gmail.com 


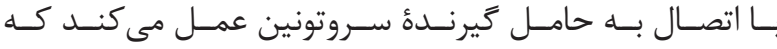

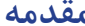

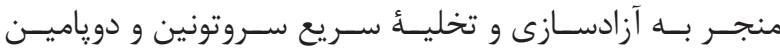

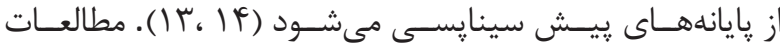

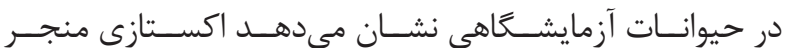

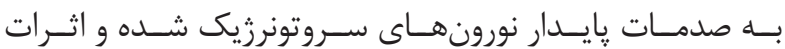

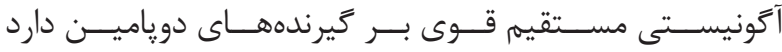

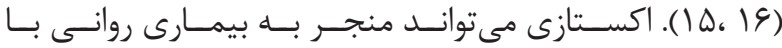

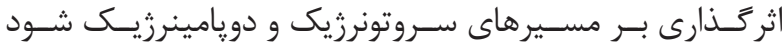

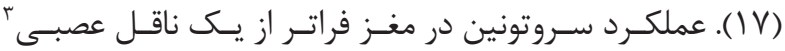

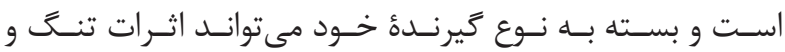

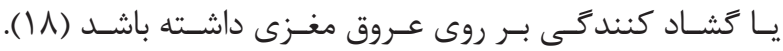

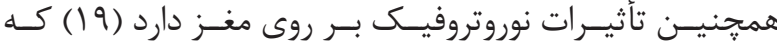

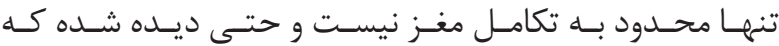

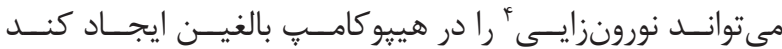

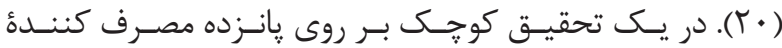

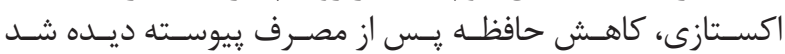

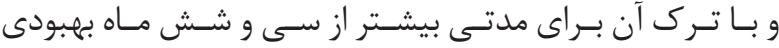

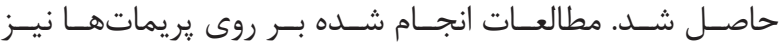

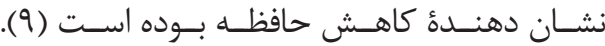

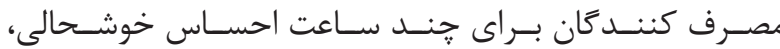

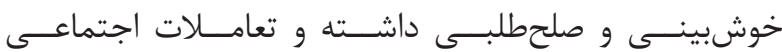

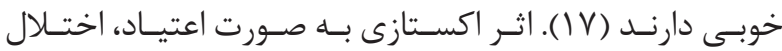

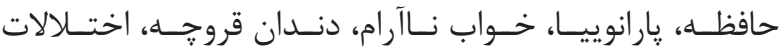

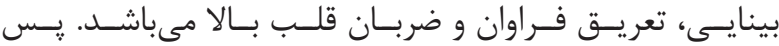

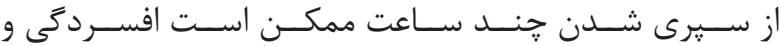

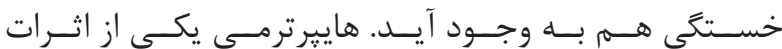

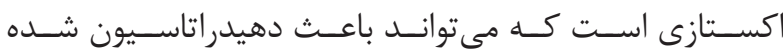

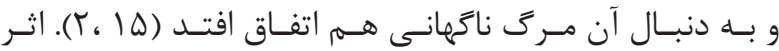

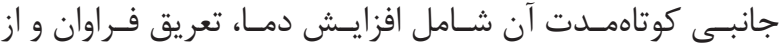

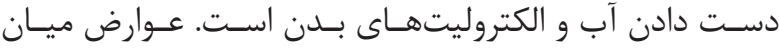

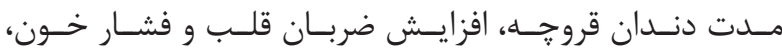

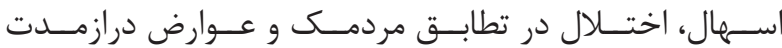

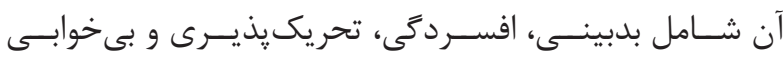

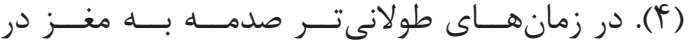

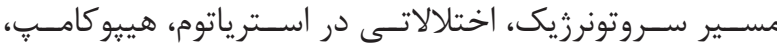

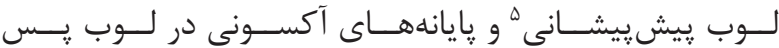

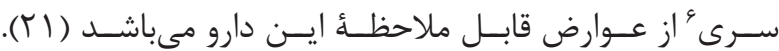

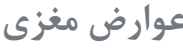

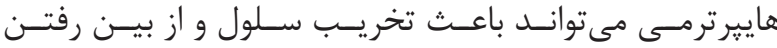

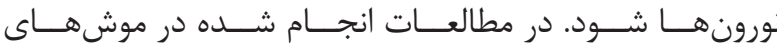

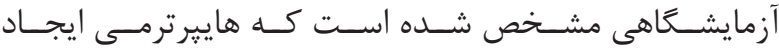

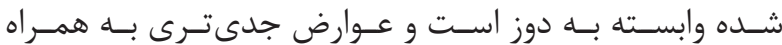

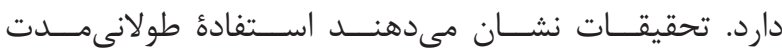

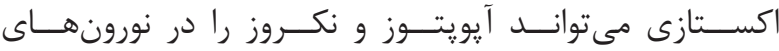

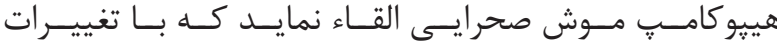

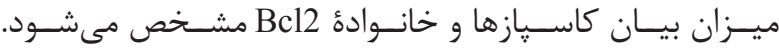

${ }^{1}$ 3,4-Methylendioxymethamphetamin, MDMA

${ }^{3}$ Neurotransmitter

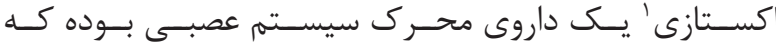

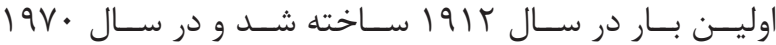

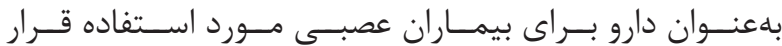

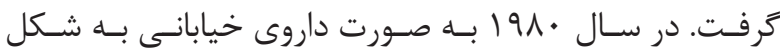

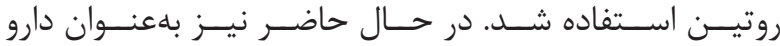

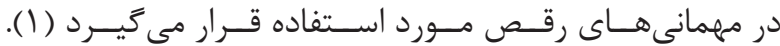

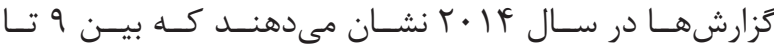

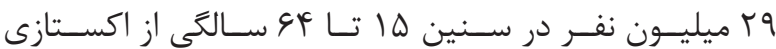

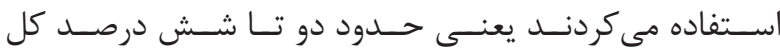

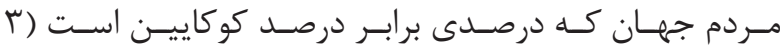

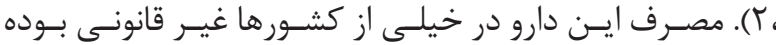

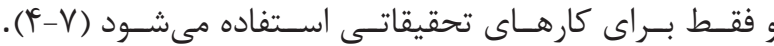

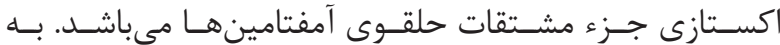

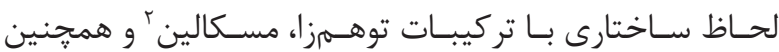

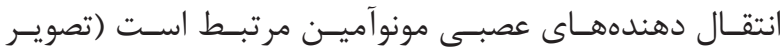

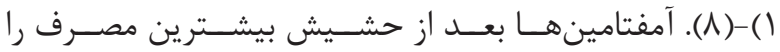

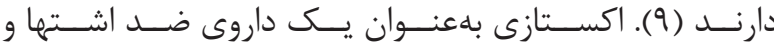

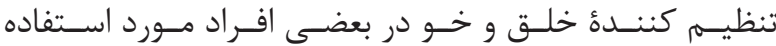

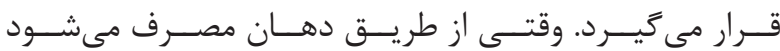

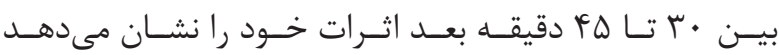

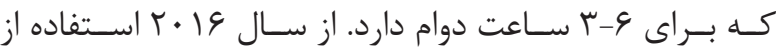

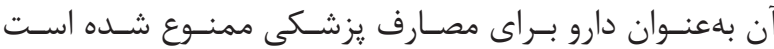

$(\mu, 96 \mathrm{~V}, 1 \cdot)$

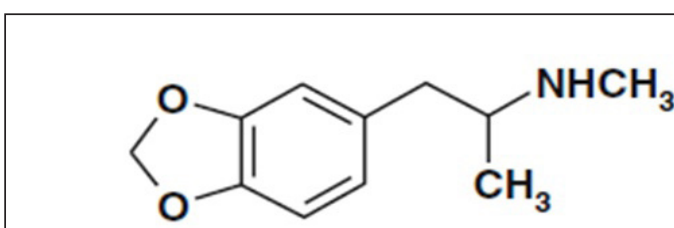

\section{3,4-Methylenodioximethamphetamine (MDMA)}

تصوير 1- ساختمان اكستازى (ᄉ).

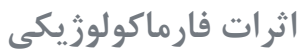

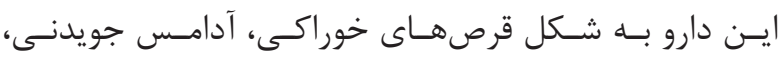

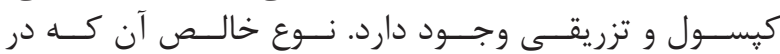

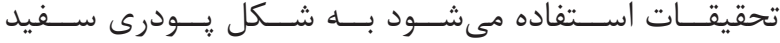

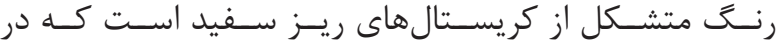

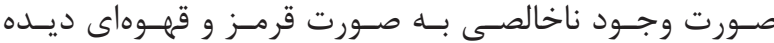
مى شـــود. مكانيسـم دقيـق علايسم ايجــاد شــده توســـ اكسـتازى بــهـ

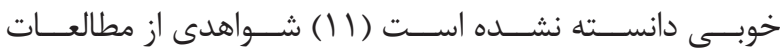

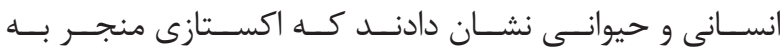

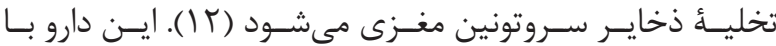

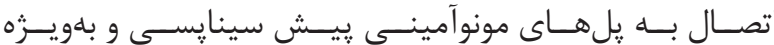




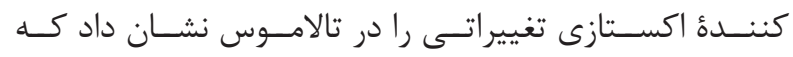

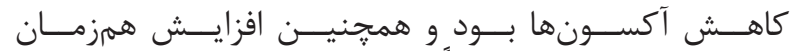

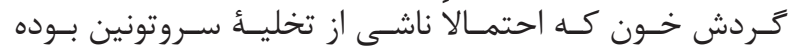

اسـت (TK).

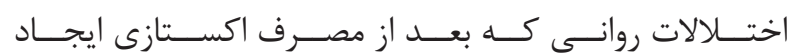

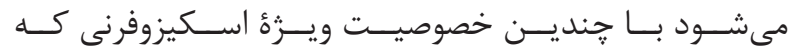

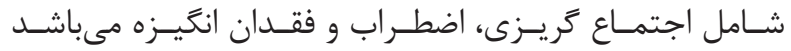

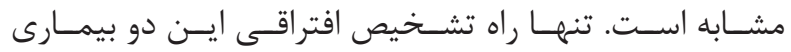

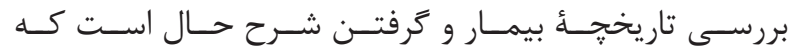

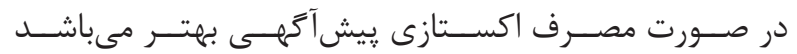

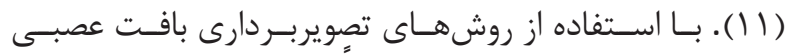

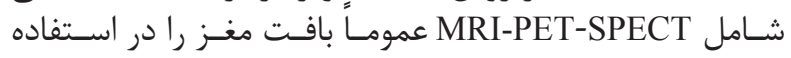

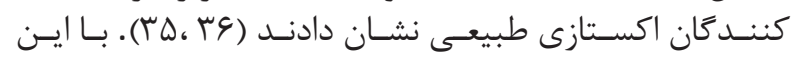

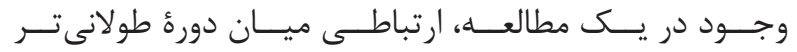

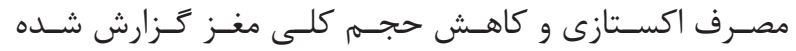

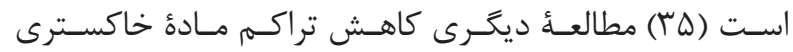

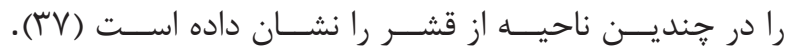

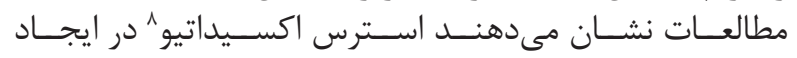

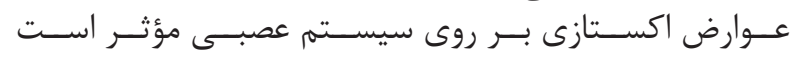

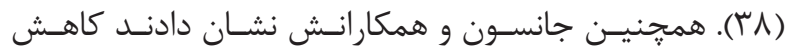

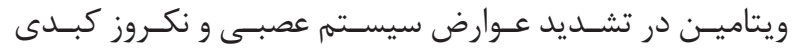

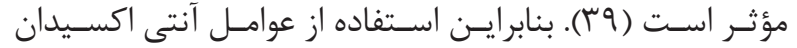

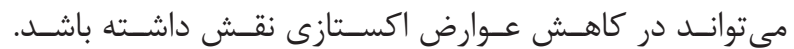

نتيجه تيرى

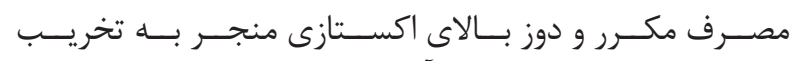

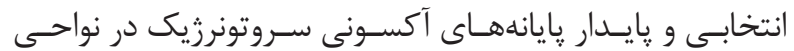

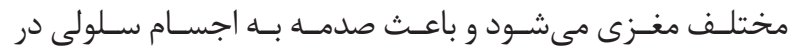

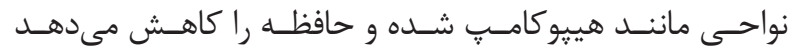

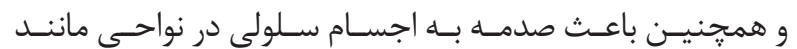

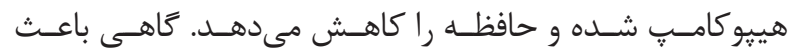

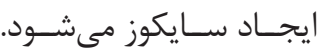

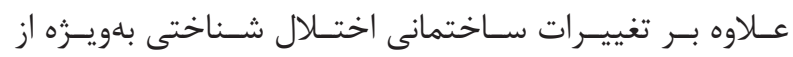

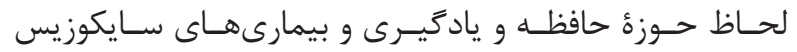

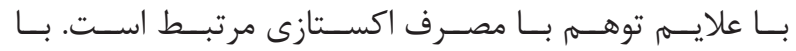

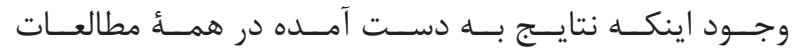

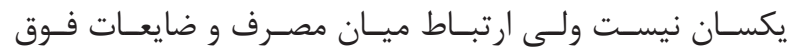

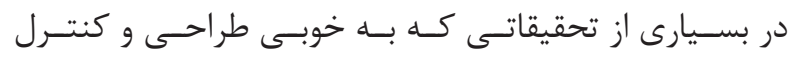

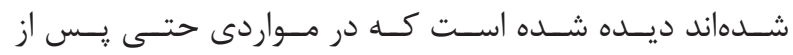

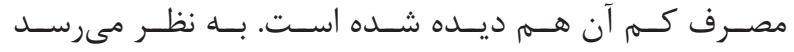

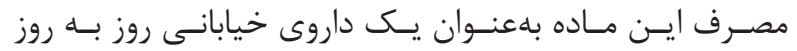

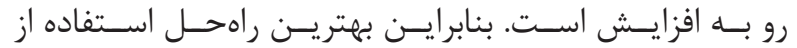

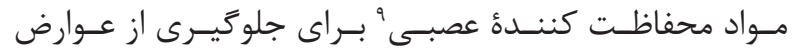

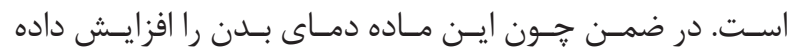

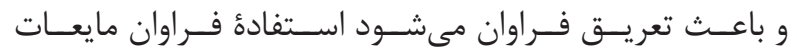

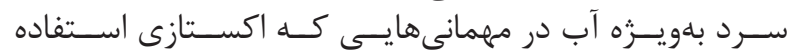

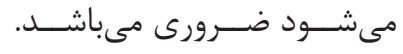

${ }^{7}$ Frontal cortex

${ }^{8}$ Oxidative stress

${ }^{9}$ Neuroprotective

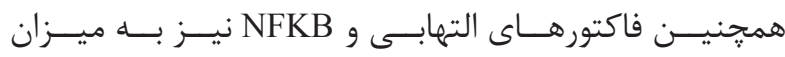

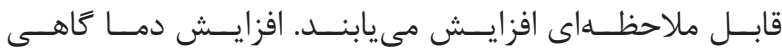

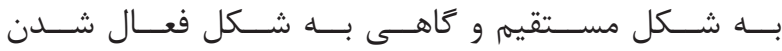

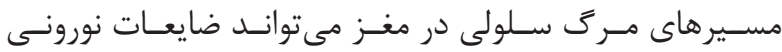

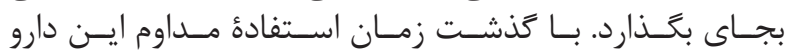

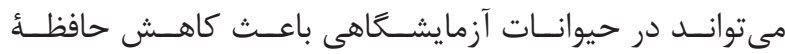

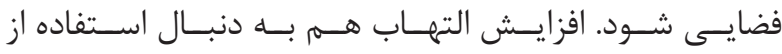

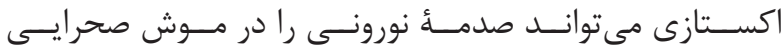

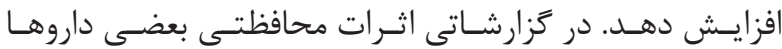

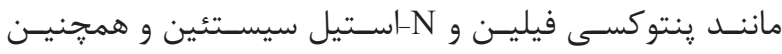

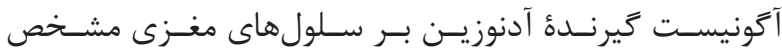

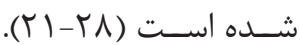

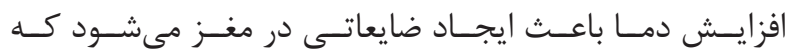

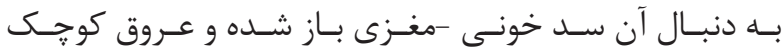

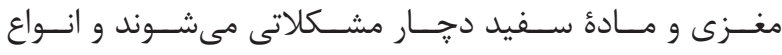

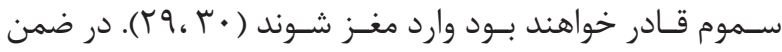

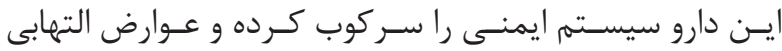

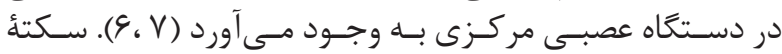

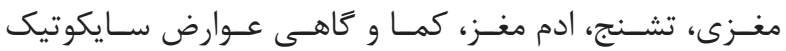

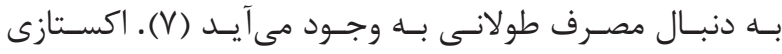

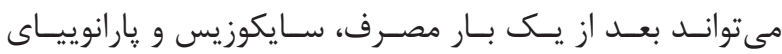

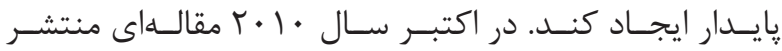

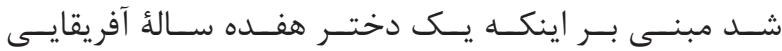

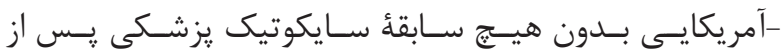

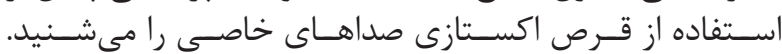

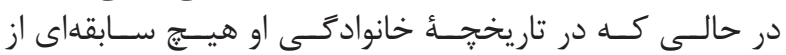

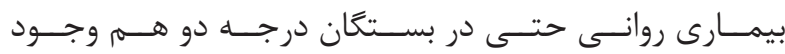

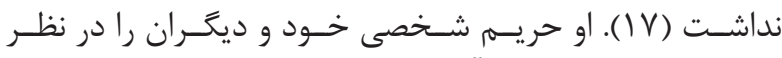

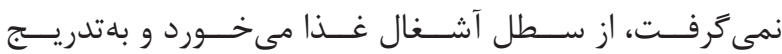

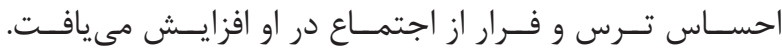

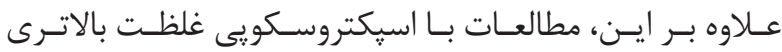

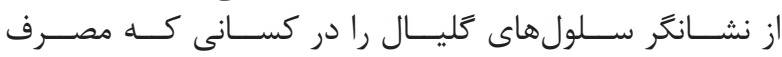

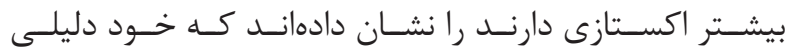

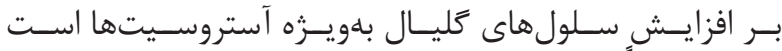

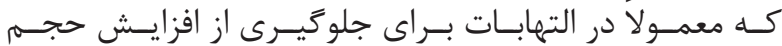

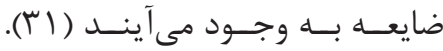

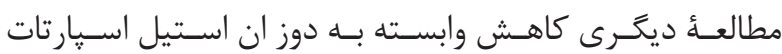

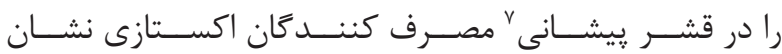

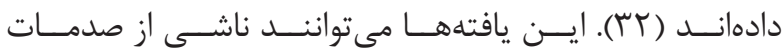

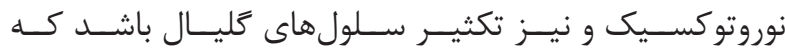

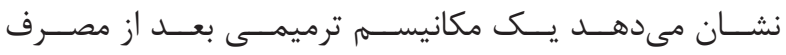

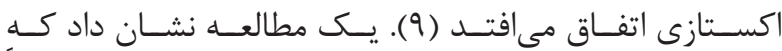

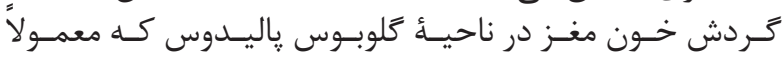

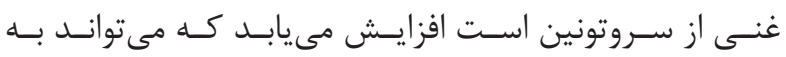

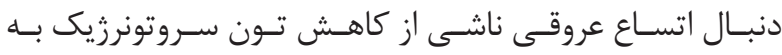

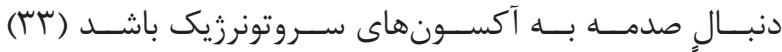

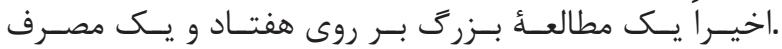


1. Organization WH. Neuroscience of psychoactive substance use and dependence: World Health Organization; 2004.

2. Smith KM, Larive LL, Romanelli F. Club drugs: methylenedioxymethamphetamine, flunitrazepam, ketamine hydrochloride, and gamma-hydroxybutyrate. Am J Health Syst Pharm. 2002; 59(11): 1067-76.

3. Malenka R, Nestler E, Hyman S. Reinforcement and addictive disorders. Molecular neuropharmacology: a foundation for clinical neuroscience. $2^{\text {nd }}$. New York: McGraw-Hill Medical. 2009. p. 364-75.

4. Meyer JS. 3,4-methylenedioxymethamphetamine (MDMA): current perspectives. Subst Abuse Rehabil. 2013; 4: 83-99.

5. Parrott AC. The potential dangers of using MDMA for psychotherapy. J Psychoactive Drugs. 2014; 46(1): 37-43.

6. Carvalho M, Carmo H, Costa VM, Capela JP, Pontes H, Remiao F, et al. Toxicity of amphetamines: an update. Arch Toxicol. 2012; 86(8): 1167-231.

7.PuertaE,AguirreN.Methylenedioxymethamphetamine (MDMA,'Ecstasy'): neurodegeneration versus neuromodulation. Pharmaceuticals. 2011; 4(7): $992-$ 1018 .

8. Capela JP, Carmo H, Remiao F, Bastos ML, Meisel A, Carvalho F. Molecular and cellular mechanisms of ecstasy-induced neurotoxicity: an overview. Mol Neurobiol. 2009; 39(3): 210-71.

9. Gouzoulis-Mayfrank E, Daumann J. Neurotoxicity of drugs of abuse-the case of methylenedioxyamphetamines (MDMA, ecstasy), and amphetamines. Dialogues Clin Neurosci. 2009; 11(3): 305-17.

10. Barceloux DG. Medical toxicology of drug abuse: synthesized chemicals and psychoactive plants. John Wiley \& Sons. 2012.

11. Van Kampen J, Katz M. Persistent psychosis after a single ingestion of 'Ecstasy'. Psychosomatics. 2001; 42(6): $525-7$.

12. Morgan MJ. Ecstasy (MDMA): a review of its possible persistent psychological effects. Psychopharmacology. 2000; 152(3): 230-48.

13. Greene SL, Kerr F, Braitberg G. Review article: amphetamines and related drugs of abuse. Emerg Med Australas. 2008; 20(5): 391-402.
14. Gouzoulis-Mayfrank E, Daumann J. Neurotoxicity of methylenedioxyamphetamines (MDMA; ecstasy) in humans: how strong is the evidence for persistent brain damage? Addiction. 2006; 101(3): 348-61.

15. Sarkar S, Schmued L. Neurotoxicity of ecstasy (MDMA): an overview. Curr Pharm Biotechnol. 2010; 11(5): 460-9.

16. WhiteS, Obradovic T, ImelK, WheatonM. The effects of methylenedioxymethamphetamine (MDMA,"Ecstasy") on monoaminergic neurotransmission in the central nervous system. Prog Neurobiol. 1996; 49(5): 455-79.

17. Patel A, Moreland T, Haq F, Siddiqui F, Mikul M, Qadir H, et al. Persistent psychosis after a single ingestion of "Ecstasy"(MDMA). Prim Care Companion CNS Disord. 2011; 13(6). doi: 10.4088/PCC.11101200.

18. Berger M, Gray JA, Roth BL. The expanded biology of serotonin. Annu Rev Med. 2009; 60: 355-66.

19. Yan W, Wilson CC, Haring JH. 5-HT1a receptors mediate the neurotrophic effect of serotonin on developing dentate granule cells. Brain Res Dev Brain Res. 1997; 98(2): 185-90.

20. Brezun J, Daszuta A. Depletion in serotonin decreases neurogenesis in the dentate gyrus and the subventricular zone of adult rats. Neuroscience. 1999; 89(4): 999-1002.

21. Kermanian F, Mehdizadeh M, Soleimani M, Ebrahimzadeh Bideskan AR, Asadi-Shekaari M, Kheradmand $\mathrm{H}$, et al. The role of adenosine receptor agonist and antagonist on Hippocampal MDMA detrimental effects; a structural and behavioral study. Metab Brain Dis. 2012; 27(4): 459-69.

22. Kermanian F, Soleimani M, Ebrahimzadeh A, Haghir $\mathrm{H}$, Mehdizadeh M. Effects of adenosine A2a receptor agonist and antagonist on hippocampal nuclear factor-kB expression preceded by MDMA toxicity. Metab Brain Dis. 2013; 28(1): 45-52.

23. Movassaghi S, Nadia Sharifi Z, Soleimani M, Joghataii MT, Hashemi M, Shafaroodi H, et al. Effect of pentoxifylline on ischemia- induced brain damage and spatial memory impairment in rat. Iran J Basic Med Sci. 2012; 15(5): 1083-90.

24. Soleimani M, Katebi M,AlizadehA, Mohammadzadeh $\mathrm{F}$, Mehdizadeh $\mathrm{M}$. The role of the A2A receptor in cell apoptosis caused by MDMA. Cell J. 2012; 14(3): 231-6.

25. Kermanian F, Soleimani M, Pourheydar B, Samzadeh- 
Kermani A, Mohammadzadeh F, Mehdizadeh M. Effects of adenosine A2a receptor agonist and antagonist on cerebellar nuclear factor-kB expression preceded by MDMA toxicity. Med J Islam Repub Iran. 2014; 28: 120.

26. Soleimani Asl S, Mousavizadeh K, Pourheydar B, Soleimani M, Rahbar E, Mehdizadeh M. Protective effects of N-acetylcysteine on 3, 4-methylenedioxymethamphetamine-induced neurotoxicity in male Sprague-Dawley rats. Metab Brain Dis. 2013; 28(4): 677-86.

27. Behroozaghdam M, Hashemi M, Javadi G, Mahdian $\mathrm{R}$, Soleimani M, Sharifi ZN, et al. The effect of MDMA and pentoxifylline drug on $\mathrm{bad} / \mathrm{bcl}-\mathrm{xl}$ gene dosage expression changes on rat liver. Journal of Paramedical Sciences. Journal of Paramedical Sciences. 2015; 6(3): 97-103.

28. Kermanian F, Mehdizadeh M, Soleimani M, Bideskan ARE, Hami J, Kheradmand $\mathrm{H}$, et al. Adenosine A2a receptors activate nuclear factor-kappa $\mathrm{B}(\mathrm{NF}-\kappa \mathrm{B})$ in rat hippocampus after exposure to different doses of MDMA. Molecular \& Cellular Toxicology. 2014; 10(1): 59-66.

29. Kousik SM, Napier TC, Carvey PM. The effects of psychostimulant drugs on blood brain barrier function and neuroinflammation. Front Pharmacol. 2012; 3: 121. doi: 10.3389/fphar.2012.00121.

30. Boyle NT, Connor TJ. Methylenedioxymethamphetamine ('Ecstasy')induced immunosuppression: a cause for concern? Br J Pharmacol. 2010; 161(1): 17-32.

31. Chang L, Ernst T, Grob CS, Poland RE. Cerebral (1)H MRS alterations in recreational 3, 4-methylenedioxymethamphetamine(MDMA, “ecstasy") users. Journal of Magnetic Resonance Imaging: JMRI. 1999; 10(4): 521-6.

32. Reneman L, Majoie CB, Flick H, den Heeten GJ. Reduced $\mathrm{N}$-acetylaspartate levels in the frontal cortex of
3, 4-methylenedioxymethamphetamine (Ecstasy) users: preliminary results. AJNR Am J Neuroradiol. 2002; 23(2): 231-7.

33. Reneman L, Majoie CB, Habraken JB, den Heeten GJ. Effects of ecstasy (MDMA) on the brain in abstinent users: initial observations with diffusion and perfusion $\mathrm{mr}$ imaging 1. Radiology. 2001; 220(3): 611-7.

34. de Win MM, Jager G, Booij J, Reneman L, Schilt $\mathrm{T}$, Lavini C, et al. Neurotoxic effects of ecstasy on the thalamus. Br J Psychiatry. 2008; 193(4): 289-96.

35. Chang L, Grob CS, Ernst T, Itti L, Mishkin FS, Jose-Melchor R, et al. Effect of ecstasy [3,4-methylenedioxymethamphetamine (MDMA)] on cerebral blood flow: a co-registered SPECT and MRI study. Psychiatry Res. 2000; 98(1): 15-28.

36. Gamma A, Buck A, Berthold T, Vollenweider FX. No difference in brain activation during cognitive performance between ecstasy (3, 4-methylenedioxymethamphetamine) users and control subjects: a [H215O]-positron emission tomography study. Journal of Clinical Psychopharmacology. 2001; 21(1): 66-71.

37. Cowan RL, Lyoo IK, Sung SM, Ahn KH, Kim MJ, Hwang J, et al. Reduced cortical gray matter density in human MDMA (Ecstasy) users: a voxel-based morphometry study. Drug Alcohol Depend. 2003; 72(3): 225-35.

38. Riezzo I, Cerretani D, Fiore C, Bello S, Centini $\mathrm{F}$, D'errico $\mathrm{S}$, et al. Enzymatic-nonenzymatic cellular antioxidant defense systems response and immunohistochemical detection of MDMA, VMAT2, HSP70, and apoptosis as biomarkers for MDMA(Ecstasy) neurotoxicity. J Neurosci Res. 2010; 88(4): 905-16.

39. Johnson EA, Shvedova AA, Kisin E, O'Callaghan JP, Kommineni C, Miller DB. d-MDMA during vitamin E deficiency: effects on dopaminergic neurotoxicity and hepatotoxicity. Brain Res. 2002; 933(2): 150-63. 